\title{
Thermodynamics of a binary monolayer of Ising dipolar particles
}

\author{
Masaru Suzuki, ${ }^{1, *}$ Ferenc Kun, ${ }^{2}$ Satoshi Yukawa, ${ }^{3}$ and Nobuyasu Ito ${ }^{1}$ \\ ${ }^{1}$ Department of Applied Physics, University of Tokyo, 7-3-1, Hongo, Bunkyo-ku, Tokyo, 113-8656, Japan \\ ${ }^{2}$ Department of Theoretical Physics, University of Debrecen, P.O. Box 5, H-4010 Debrecen, Hungary \\ ${ }^{3}$ Department of Earth and Space Science, Graduate School of Science, Osaka University,1-1 Machikaneyama-cho, \\ Toyonaka-shi, Osaka 560-0043, Japan
}

(Received 29 May 2007; published 15 November 2007)

\begin{abstract}
Thermodynamic behavior of a binary monolayer of Ising dipolar particles is studied using numerical simulation. The thermal equilibrium states of the system under the canonical ensemble are observed. The boundary of gas-liquid phase is determined from the power law growth of critical clusters. But that temperature is slightly lower than that of solidification. So it means that this system does not have a liquid phase. This system is also studied theoretically using virial expansion. The coefficients obtained from standard virial expansion, however, do not show any phase transition. An improvement of the virial expansion is also given by taking tetragonal local structures of alternate types of particles into consideration. Such structures are observed within critical clusters of our simulation. The thermodynamic state equation obtained from the improved virial expansion agrees well with the simulation result, and this expansion also shows that the critical point is almost at the same temperature with solidification. These results suggest that not simply the strength of attractive force by the dipole interaction but a typical configuration caused by the binary condition of this system plays a dominant role in phase transitions.
\end{abstract}

DOI: 10.1103/PhysRevE.76.051116

PACS number(s): 05.70.-a, 83.10.Pp, 75.50.Mm, 61.20.Gy

\section{INTRODUCTION}

Behavior of the two- and three-dimensional particle systems with hard (or soft) core interaction and embedded dipolar moment has been intensely investigated recently [1-15]. It was found that such systems show richer ground state structure and thermal behavior than simple particle systems. For example, the gas-liquid transition of the dipolar particle system is much different from that of Lennard-Jones and other simple particle systems [2,3,5,6,9], or there is completely no liquid phase $[4,16]$. This is because anisotropic chaining with local head-to-tail alignment occurs and such chains prevent normal liquid condensation of particles. The ground state structures are also interesting. The ground state of the hexagonal-closed-packed lattice has shown to have a macrovortex alignment of dipolar moment, while microvortex and antiparallel alignment have the same lowest energy for a square lattice. And at low density, a configuration with concentric layers of rings which looks like a "hollow vortex" has the lowest energy $[8,10]$.

A monolayer of a dipolar particle model in which the dipole orientation of each particle is fixed to be up or down is named the Ising dipolar particle (IDP) model $[14,15]$. Although anisotropy of particle interaction is eliminated, this system still shows interesting pattern formation. Experimental implementations of a binary monolayer of Ising dipolar particles were proposed: binary colloids composed of two sorts of $\mu \mathrm{m}$-sized dielectric particles suspended in water and subjected to an ac electric field [12], or floating cork disk with small magnet [13]. This Ising dipolar system is also investigated theoretically by numerical simulation [14]. It has been shown that these systems show a variety of struc-

\footnotetext{
*suzuki@serow.t.u-tokyo.ac.jp
}

tures like chains, rings, and branched structures in dilute condition, as well as various types of crystalline structures at high density depending on the parameters like relative concentration and relative dipole moment of two components. Detailed analysis of the aggregation process showed a fractal behavior of growing clusters, and fractal dimension is consistent with the widely observed value of diffusion-limited cluster-cluster aggregation in other particle systems [17]. Further study showed the kinetic characteristics of aggregation according to a dynamic scaling theory and estimated the dynamical exponent, which is much smaller than other dipolar systems [15].

Such observed aggregates like chains have, however, rather fragile structure, which has shown to have only a small energy barrier to change its shape [17], so the existence of thermal noise may change the aggregation process. Furthermore, the thermodynamic phase diagram of this system is not known yet. In this paper, results of our numerical study of the thermodynamic properties of the binary monolayer of an Ising dipolar system, and also a theoretical approach to reproduce the thermodynamic phases of this system, are reported.

The outline of this paper is as follows. The model and method of our simulation is introduced in Sec. II. In Sec. III simulation results mainly around critical condensation and data analysis to determine the phase boundary are shown. In Sec. IV those results are analytically examined by using a perturbative method. An improved virial expansion taking local structures into consideration is proposed. Section V is the summary and, finally, in Sec. VI we discuss how dipolar interaction and binary condition affect the phase transitions of this system.

\section{MODEL AND METHOD}

Our simulation model consists of $N$ Ising dipole particles in a square box of sidelength $L$ with periodic boundary con- 
dition. All the particles have the same mass $m$. Each particle has spherical elastic volume of radius $R$ and a pointlike dipole moment at the center. The dipole moment of each particle is fixed during the time evolution and it is perpendicular to the plane of motion pointing either upward or downward. So the pair potential between $i$ th particle and $j$ th particle with distance $r_{i j}$ is

$$
\Phi\left(r_{i j}\right)=\Phi^{\mathrm{pp}}\left(r_{i j}\right)+\frac{\mu_{i} \mu_{j}}{r^{3}},
$$

where $\mu_{i}$ and $\mu_{j}$ denote the dipole moments of $i$ th and $j$ th particles, respectively. The cutoff distance is taken to be $8 d$ here, where $d(=2 R)$ denotes the diameter of the particles. We considered that, though a long-range interacting system is treated, the error caused by this interaction cutoff is very small as a shielding effect works well in our neutral condition. Actually we have calculated the energy per particle of a crystalline configuration of square lattice (with lattice constant $d$ ) and found the error by this cutoff length is about $0.12 \%$. It is also pointed out in [18] that there is no substantial effect of cutoff for the ground state configurations of ordinal dipolar particles with the cutoff length larger than $8 d$. The absolute strength of every dipole moment is $\mu_{0}$, so $\mu_{i}$ $=\mu_{0}$ (upward) or $\mu_{i}=-\mu_{0}$ (downward). Therefore, the interaction is repulsive between the particles with same moment and attractive between those with opposite moment. In the following, we treat only the case that the numbers of particles of both dipole types are equal. $\Phi^{\mathrm{pp}}$ denotes a core potential. Though particles with hard core potential are interesting, for the efficiency of the particle dynamics simulation, small overlap of particles is assumed using the Hertzian contact potential:

$$
\Phi^{\mathrm{pp}}\left(r_{i j}\right)= \begin{cases}\frac{2}{5} k_{\mathrm{pp}}\left(d-r_{i j}\right)^{5 / 2} & \left(r_{i j}<d\right) \\ 0 & \left(r_{i j}>d\right),\end{cases}
$$

where the value of elastic constant $k_{\mathrm{pp}}$ is taken to be $k_{\mathrm{pp}}$ $=400 \sqrt{2} \mu_{0}^{2} / d^{5.5}$ in our simulation. The core repulsion between two particles balances with dipolar interaction at a distance of about $0.97 d$.

We execute a series of simulations under the canonical $(T N V)$ ensemble. Instead of $V$, hereafter we use the density defined as coverage $\phi \equiv N \pi R^{2} / V$, and the Boltzmann constant is set using the binding energy of two particles as $k_{B}$ $=2 \mu_{0}^{2} d^{-3}$.

The isothermal particle dynamics method [19] is used for temperature control. In this method, the total kinetic energy of the system is conserved during the time evolution. Started from a particle configuration whose kinetic energy is equal to $N K_{B} T$, the configuration is updated by the leap-frog scheme with a time step $\Delta t$, that is,

$$
\begin{gathered}
\boldsymbol{r}_{i}^{\prime}=\boldsymbol{r}_{i}^{t}+\frac{\Delta t}{2} \boldsymbol{v}_{i}^{t}, \\
\boldsymbol{v}_{i}^{t+\Delta t}=\boldsymbol{v}_{i}^{t}+\Delta t \frac{1}{m} \sum_{i \neq j}^{N} \boldsymbol{F}\left(r_{i j}^{\prime}\right)-\chi \boldsymbol{v}_{i}^{t},
\end{gathered}
$$

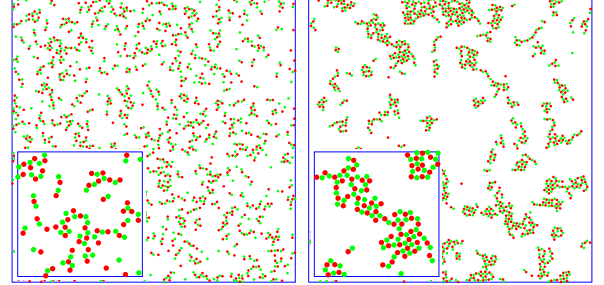

FIG. 1. (Color online) Snapshots from the equilibrium state at $\phi=0.125, T=0.100$ (left) and at $\phi=0.125, T=0.060$ (right) are shown. Many clusters with finite size move around, instead of aggregating into a large cluster. Each inset shows a snapshot focused on some typical clusters.

$$
\boldsymbol{r}_{i}^{t+\Delta t}=\boldsymbol{r}_{i}^{\prime}+\frac{\Delta t}{2} \boldsymbol{v}_{i}^{t+\Delta t}
$$

where an artificial viscosity coefficient $\chi$ is determined at every step to satisfy $\frac{1}{2} m \sum_{i=1}^{N}\left(v_{i}^{t+\Delta t}\right)^{2}=\frac{1}{2} m \sum_{i=1}^{N}\left(v_{i}^{t}\right)^{2}$. So it is calculated as

$$
\chi=\frac{1}{v^{2}}\left(\overline{v^{2}}+\overline{\boldsymbol{v} \cdot \boldsymbol{f}} \Delta t-\sqrt{\overline{v^{2} 2}+\left(\overline{\boldsymbol{v} \cdot \boldsymbol{f}^{2}}-\overline{f^{2} v^{2}}\right) \Delta t^{2}}\right) \quad(\boldsymbol{f} \equiv \boldsymbol{F} / m) .
$$

The bars over the variables in the equation denote the averaged values of all particles like $\overline{v^{2}}=\sum_{i=1}^{N} v_{i}^{2} / N$.

Three kinds of initial particle locations are prepared and it is confirmed that these three simulations reach the same result after thermalization steps: (a) Uniformly random location without particle overlap, (b) square lattice location where both types of particles are alternately located, and (c) mixture of (a) and (b) where $\frac{1}{2} N$ particles construct a square lattice cluster and others are located randomly uniform around that cluster.

\section{RESULT}

At the region of high temperature and relatively low density, the system behaves like ideal gas. Dimerized pairs of particles with opposite moments appear at the temperature around 0.5. As temperature is decreased, particles begin to aggregate to clusters; however, the growth of the cluster size is so moderate that even at the much lower temperature as $T \sim 0.060$ clusters remain finite and move around in the system (Fig. 1).

The average cluster size $\bar{s}$ is measured at each temperature and density. Here a cluster is defined as follows: Two particles are in the same cluster if they are linked by more than one path of contacting pairs. The average cluster size (the number of particles in a cluster) is defined by

$$
\bar{s} \equiv \frac{\sum_{s} s^{2} n(s)}{\sum_{s} s n(s)},
$$

where $s$ is the size of a cluster and $n(s)$ is the number of the clusters with size $s$. 


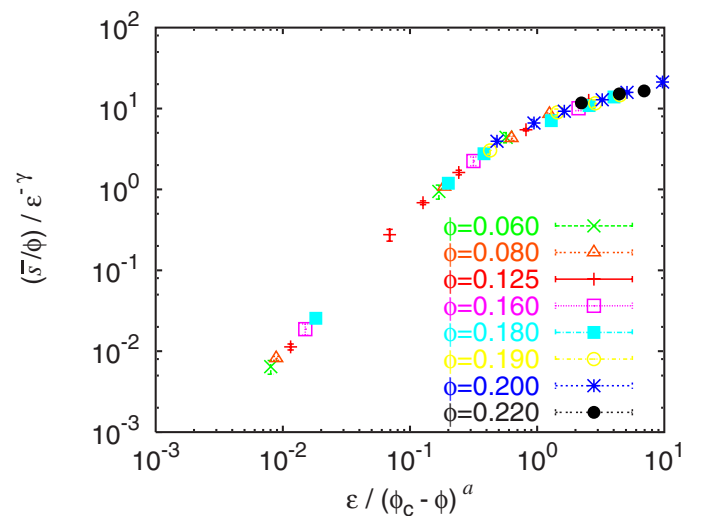

FIG. 2. (Color online) Scaling plot of the average cluster size with Eq. (6) is shown.

It was found that the average cluster size shows a critical behavior as $\bar{s} \sim\left(T-T_{c}\right)^{-\gamma}$, with a certain critical temperature $T_{c}$, and $\bar{s} / \phi \sim\left(\phi_{c}-\phi\right)^{-\gamma^{\prime}}$, with a certain critical density $\phi_{c}$. So the critical analysis of liquid condensation is done assuming a scaling form

$$
\bar{s} / \phi \sim \epsilon^{-\gamma} f_{s}\left[\epsilon /\left(\phi_{c}-\phi\right)^{a}\right],
$$

where $\epsilon=\left(T-T_{c}\right) / T_{c}$ and $f_{s}$ is a scaling function. Figure 2 shows the scaling plot with Eq. (6). All points fall onto a single curve when the following critical parameters are used:

$$
\begin{gathered}
T_{c}=0.05790 \pm 0.00005, \quad \phi_{c}=0.275 \pm 0.005, \\
\gamma=1.76 \pm 0.01, \quad \text { and } \quad a=1.00 \pm 0.01 .
\end{gathered}
$$

The critical behavior of the correlation length was also investigated. The correlation length $\xi$ was defined to be

$$
\xi^{2} \equiv \frac{\sum_{s} R_{g}^{2} s^{2} n(s)}{\sum_{s} s^{2} n(s)},
$$

where $R_{g}$ is the gyration radius of a cluster, which is defined to be

$$
R_{g}^{2}=\frac{1}{s(s-1)} \sum_{i<j}\left(r_{i}-r_{j}\right)^{2} .
$$

The scaling form of $\xi$ was assumed to be

$$
\xi \sim \epsilon^{-\nu} f_{\xi}\left[\epsilon /\left(\phi_{c}-\phi\right)^{b}\right] .
$$

Figure 3 shows the scaling plot of $\xi$ with Eq. (10) by setting

$$
\nu=0.99 \pm 0.01, \quad b=1.05 \pm 0.05 \text {. }
$$

We are confident that the finite size of the system has very little effect on those plots in Figs. 3 and 2, by comparing the results of systems with $N=1600, N=3200$, and $N=6400$.

Though the critical point of liquid condensation is estimated from the critical phenomena, a different kind of change happens at the temperature slightly higher than $T_{c}$. At that temperature most particles form a single compact cluster with ordered distribution of alternating components as illus-

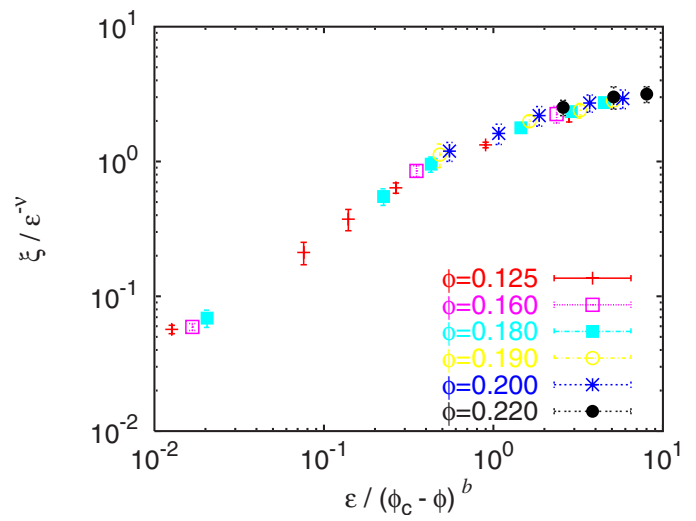

FIG. 3. (Color online) Scaling plot of the correlation length with Eq. (10) is shown.

trated in Fig. 4, and that is a solid state cluster.

To determine the solidification temperature $T_{S}$, an orientational order parameter $\phi_{4}[20]$,

$$
\phi_{4}=\left|\frac{1}{N} \sum_{\langle i, j\rangle} \exp \left(4 \mathbf{i} \theta_{i j}\right)\right|, \quad\left(\cos \theta_{i j}, \sin \theta_{i j}\right) \equiv \frac{\boldsymbol{r}_{i j}}{r_{i j}},
$$

is calculated, where $\langle i, j\rangle$ denotes all the pairs of neighboring particles and $\mathbf{i}$ is the imaginary unit. The maximum value of $\phi_{4}$, which corresponds to a perfect square lattice order, is $\phi_{4}=2$.

The value of $\phi_{4}$ in equilibrium state was measured for the largest cluster and the average cluster size was also calculated. As plotted in Fig. 5 discontinuous change of the average cluster size occurs at certain temperature. Simultaneously, the largest cluster comes to have order of $\phi_{4}$. The temperature of solidification was extrapolated from the results of some finite sized systems in the range of $N$ $=800-6400$, and then it was estimated to be

$$
T_{s}=0.0585 \pm 0.0001 \text { at } \phi=0.25
$$

(and $T_{s}=0.0576$ at $\phi=0.125$ ).

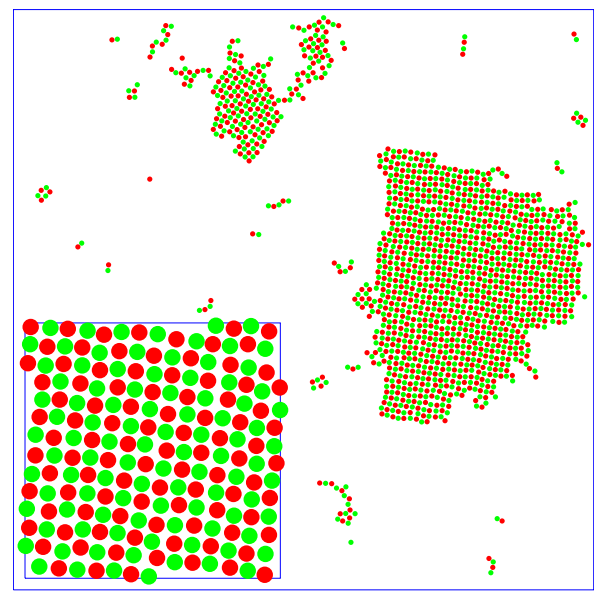

FIG. 4. (Color online) Formation of a solid cluster with square lattice order of alternating particles at $N=1600, \phi=0.125$, and $T$ $=0.055$. The inset shows an inner part of the largest cluster. 


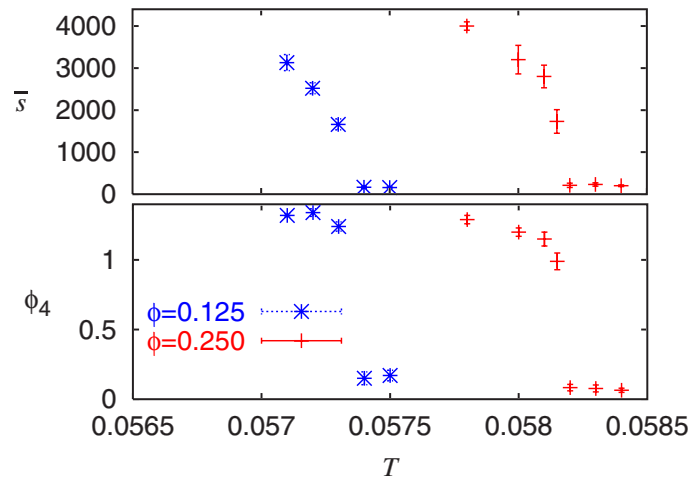

FIG. 5. (Color online) Average cluster size (above) and the orientational order $\phi_{4}$ (below) around the solidification are shown. Each plot is the time average after the system reached equilibrium state.

The curious fact that should be emphasized is the critical temperature of gas-liquid transition estimated to be $T_{c}$ is lower than that of solidification $T_{s}$. It suggests that the critical phenomenon is surely observed; however, this system does not have liquid phase.

Based on the analysis above, Fig. 6 shows the schematic drawing of the $\phi-T$ phase diagram. The critical point of the gas-liquid phase transition should exist as plotted in the diagram, but it is actually hidden in the region of the fluid-solid phase. The phase boundary at relatively high density is also shown in that diagram, based on another series of our simulations.

\section{VIRIAL EXPANSION ANALYSIS}

The most characteristic feature of this system is, as it is shown in the preceding section, that the gas-liquid critical point is close to the solidification temperature, and that the liquid phase vanishes. This feature is reproduced by an improved virial expansion considering local structures of Ising dipolar particles, although a naive application of the standard Mayer-Mayer expansion fails to catch such behaviors, as we will see in the following.

In the standard Mayer-Mayer expansion, each Boltzmann weight of interaction between two particles is expanded around the high temperature with a small parameter

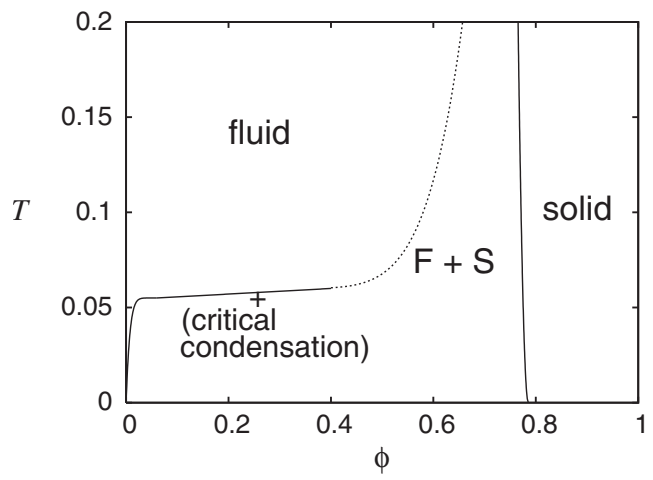

FIG. 6. Outline of the $\phi-T$ phase diagram.

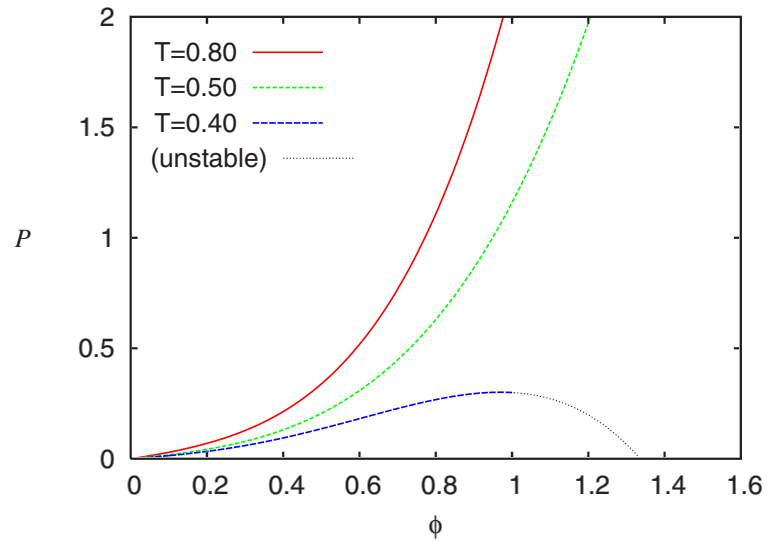

FIG. 7. (Color online) State equation curves calculated by virial expansion up to third order are displayed.

$f\left(r_{12}, T\right)=\exp \left[-\Phi\left(r_{12}\right) / k_{B} T\right]^{-1}$, which is named the Mayer function, and then the whole interaction part of the partition function is expanded with this $f$ [21]. As a consequence of the Mayer expansion, the state equation is expressed as

$$
P(\phi)=k_{B} T\left[\frac{\phi}{\pi R^{2}}-\sum_{k} \frac{k}{k+1} \beta_{k}\left(\frac{\phi}{\pi R^{2}}\right)^{k+1}\right],
$$

and the coefficients $\beta_{k}(k=1,2, \ldots)$ are calculated as

$$
\beta_{1}=\frac{1}{2^{2}} \sum_{i, j= \pm 1} \int f_{i j}\left(r_{12}\right) d r_{12}
$$

$$
\begin{aligned}
\beta_{2}= & \frac{1}{2 !} \frac{1}{2^{3}} \sum_{i, j, l= \pm 1} \int f_{i j}\left(r_{12}\right) f_{j l}\left(r_{23}\right) f_{l i}\left(r_{31}\right) d \boldsymbol{r}_{12} d \boldsymbol{r}_{23}, \\
\beta_{3}= & \frac{1}{3 !} \frac{1}{2^{4}} \sum_{i, j, l, m= \pm 1} \int f_{i j}\left(r_{12}\right) f_{j l}\left(r_{23}\right) f_{l m}\left(r_{34}\right) f_{m i}\left(r_{41}\right) \\
& \times\left[3+6 f_{i l}\left(r_{13}\right)+f_{i l}\left(r_{13}\right) f_{j m}\left(r_{24}\right)\right] d \boldsymbol{r}_{12} d \boldsymbol{r}_{23} d \boldsymbol{r}_{34},
\end{aligned}
$$

where the suffixes $i, j, l$, and $m$ of the Mayer function $f$ denote the dipole direction of each particle in a pair, and they take either value of +1 or -1 corresponding to $+\mu_{0}$ or $-\mu_{0}$ dipole moment. So the Mayer function $f_{i j}$ can be written as

TABLE I. Numerically calculated values of the virial coefficients are shown.

\begin{tabular}{lccc}
\hline \hline$T$ & $\beta_{1}$ & $\beta_{2}$ & $\beta_{3}$ \\
\hline 0.8 & -7.91 & -30.4 & -171 \\
0.5 & -5.66 & -54.2 & -71.3 \\
0.4 & -3.29 & -90.7 & 219 \\
0.2 & 21.9 & $-1.23 \times 10^{3}$ & $2.98 \times 10^{4}$ \\
0.1 & 275 & $-9.39 \times 10^{4}$ & $1.43 \times 10^{7}$ \\
0.04 & $2.28 \times 10^{5}$ & $-7.26 \times 10^{10}$ & $3.16 \times 10^{17}$ \\
0.02 & $3.79 \times 10^{10}$ & $-2.13 \times 10^{21}$ & $1.55 \times 10^{38}$ \\
\hline \hline
\end{tabular}




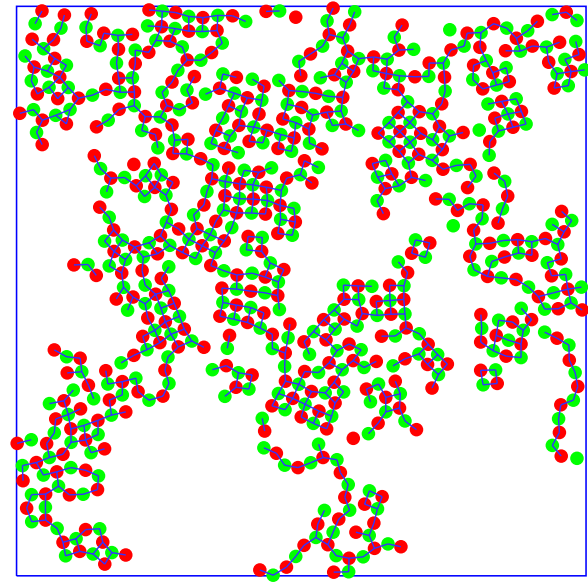

FIG. 8. (Color online) Snapshot focused on the inner part of a cluster of critical region is shown. $(\phi=0.125, T=0.0574$, and $N$ $=6400$.)

$$
f_{i j}(r)=\exp \left[-\left(\Phi^{\mathrm{pp}}(r)+i j \frac{\mu_{0}^{2}}{r^{3}}\right) / k_{B} T\right]-1 .
$$

For simplicity, the hard core potential is used for $\Phi^{p p}$.

The values of virial coefficients at various temperatures are numerically calculated up to third order $(k=3)$ which is given in Table I, and some state equation curves are shown in Fig. 7. The condition that gas-liquid phase separation happens is that Eq. (14) shows a van der Waals like loop, i.e., a tangential bifurcation of roots of $d P(\phi) / d \phi=0$ happens. But the expansion up to the third-order terms of this system does not give any phase transition because either (1) $P(\phi)$ behaves as a monotonic increasing function in $\phi>0$ or (2) after increasing with $\phi$, an unstable decreasing curve appears. The unstable curve here corresponds to a local maximum of free energy. In both cases there is no more than one root of $P^{\prime}=0$ in $\phi>0$.

This standard virial approximation result disagrees with the simulation result because this system never has a phase transition according to this expansion and, furthermore, the state equation curves are quantitatively far from our simulation result (see Fig. 10).

This obvious gap between the virial expansion and the simulation result will come from a binary condition of this system. In this binary system where the interactions are neutralized from the mean field view $\left(N_{\uparrow} \mu_{\uparrow}+N_{\downarrow} \mu_{\downarrow}=0\right)$, the energy drastically changes depending on local configurations of particles, even though the density as a whole system is not changed.

Figure 8 shows inner configurations of a critical cluster. Critical clusters in this system locally have tetragonal configurations and neighboring particles have opposite dipole moment. This is because, near the condensation point, the temperature is so much lower than the binding energy of antiparallel moments that a specific local configuration of energy minimum locally appears. So the liquid should be a mixture of local clusters with such tetragonal configurations. The virial expansion analysis treated above seems to fail to

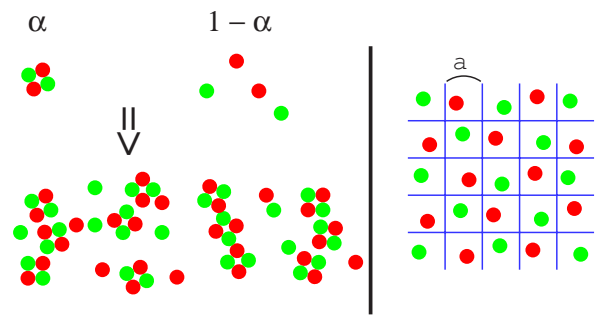

FIG. 9. (Color online) Left, schematic drawing of the tetramer model and aggregation under this model (mixture of tetramers and monomers). Right, cell divided model for solidification.

accurately estimate such specific local structure.

In order to improve the virial expansion analysis, another parameter is introduced. This time, some particles in the system, whose ratio to the whole system is $\alpha$, are assumed to form $\frac{1}{4} \alpha N$ tetramers, and other $(1-\alpha) N$ particles remain as monomers. Each tetramer which consists of both types of particles two by two has an alternately positioned tetragonal configuration with contact at distance $d$, and a tetramer does not change its shape (Fig. 9, left). Not a dimer but a tetramer structure is adopted because we assume that such a simple configuration as a dimer can be sufficiently estimated by the standard virial expansion analysis tried above.

Under this assumption, we again calculate the values of the virial coefficients up to second order as shown in Table II. The coefficients $\beta_{k l}(k=1,2, \ldots, l=0, \ldots, k+1)$ are calculated as

$$
\begin{gathered}
\beta_{1 l}=\frac{1}{2^{2-l} 4^{l}} \sum_{\substack{i, j= \pm 1, t, \delta_{i t}+\delta_{j t}=l}} \int f_{i j}\left(r_{12}\right) d \boldsymbol{r}_{12}, \\
\beta_{2 l}=\frac{1}{2 !} \frac{1}{2^{3-l} 4^{l}} \sum_{\substack{i, j, m= \pm 1, t, \delta_{i t^{+}}+\delta_{j t}+\delta_{m t}=l}} \int f_{i j}\left(\boldsymbol{r}_{12}\right) f_{j m}\left(\boldsymbol{r}_{23}\right) f_{m i}\left(\boldsymbol{r}_{31}\right) d \boldsymbol{r}_{12} d \boldsymbol{r}_{23},
\end{gathered}
$$

where the suffixes $i, j$, and $m$ of $f$ denote the type of each component in a pair, which takes either $+1,-1$, or $t$ corresponding to a particle with $\mu_{0}$, one with $-\mu_{0}$, or a tetramer, respectively. And the suffix $l$ in $\beta$ denotes the number of tetramer components in an integration. Thus

$$
\begin{gathered}
f_{i j}\left(\boldsymbol{r}_{12}\right)=-1+\frac{1}{(2 \pi)^{2}} \int \exp \left\{-\left[\Phi_{i j}^{\mathrm{pp}}\left(\boldsymbol{r}_{12}, \theta_{1}, \theta_{2}\right)\right.\right. \\
\left.\left.+\Phi_{i j}^{\mathrm{dd}}\left(\boldsymbol{r}_{12}, \theta_{1}, \theta_{2}\right)\right] / k_{B} T\right\} d \theta_{1} d \theta_{2}, \\
\Phi_{i j}^{\mathrm{pp}}\left(\boldsymbol{r}_{12}, \theta_{1}, \theta_{2}\right)=\sum_{i^{\prime}=1}^{4} \sum_{j^{\prime}=1}^{4} \Phi^{\mathrm{pp}}\left[\mid \widetilde{r}_{12}+\delta_{i t} \widetilde{r}_{0}\left(i^{\prime}, \theta_{1}\right)\right. \\
\left.-\delta_{j t} \widetilde{r}_{0}\left(j^{\prime}, \theta_{2}\right) \mid\right],
\end{gathered}
$$


TABLE II. The values of the virial coefficients of the tetramer model are shown.

\begin{tabular}{lccccccc}
\hline \hline$T$ & $\beta_{10}$ & $\beta_{11}$ & $\beta_{12}$ & $\beta_{20}$ & $\beta_{21}$ & $\beta_{22}$ & $\beta_{23}$ \\
\hline 0.3 & -2.55 & -13.9 & -3.59 & -164 & -48.2 & -66.1 & -15.2 \\
0.2 & 12.7 & -11.1 & -3.24 & -781 & 13.9 & -50.6 & -13.0 \\
0.1 & 191 & 11.1 & -0.41 & $-4.73 \times 10^{4}$ & -9.95 & -50.8 & -7.04 \\
0.08 & 512 & 39.5 & 3.41 & $-3.24 \times 10^{5}$ & $5.15 \times 10^{3}$ & -143 & -21.0 \\
0.074 & 802 & 57.8 & 6.03 & $-7.99 \times 10^{5}$ & $2.09 \times 10^{4}$ & -54.4 & -30.7 \\
0.073 & 865 & 61.7 & 6.62 & $-9.31 \times 10^{5}$ & $2.63 \times 10^{4}$ & -3.42 & -31.6 \\
\hline \hline
\end{tabular}

$$
\begin{aligned}
& \Phi_{i j}^{\mathrm{dd}}\left(\boldsymbol{r}_{12}, \theta_{1}, \theta_{2}\right) \\
& =\sum_{i^{\prime}=1}^{4} \sum_{j^{\prime}=1}^{4} \frac{\mu_{0}^{2}\left[\frac{1}{4} i \delta_{|i| 1}+(-1)^{i^{\prime}} \delta_{i t}\right]\left[\frac{1}{4} j \delta_{|j| 1}+(-1)^{j^{\prime}} \delta_{j t}\right]}{\left|\widetilde{r}_{12}+\delta_{i t} \widetilde{r}_{0}\left(i^{\prime}, \theta_{1}\right)-\delta_{j t} \widetilde{r}_{0}\left(j^{\prime}, \theta_{2}\right)\right|^{3}}, \\
& \widetilde{r}_{0}\left(i^{\prime}, \theta\right)=\sqrt{2} R \exp \left[\left(\frac{\pi}{2} i^{\prime}+\theta\right) \mathbf{i}\right], \quad \widetilde{r}_{12}=\boldsymbol{r}_{12} \cdot\left(\begin{array}{l}
1 \\
\mathbf{i}
\end{array}\right),
\end{aligned}
$$

where $\mathbf{i}$ is the imaginary unit, $\delta$ is the Kronecher delta, and $\theta$ denotes the orientation of a tetramer.

First the optimized value of $\alpha$ which minimizes the free energy [denoted as $\left.\alpha^{*}(\phi, T)\right]$ is estimated for every $(T, \phi)$, and then the pressure is calculated as

$$
\begin{aligned}
P= & k_{B} T\left[\left(1-\frac{3}{4} \alpha^{*}\right) \frac{\phi}{\pi R^{2}}-\sum_{k=1} \sum_{l=0}^{k+1}\left(1-\alpha^{*}\right)^{k+1-l}\right. \\
& \left.\times\left(\alpha^{*}\right)^{l} \frac{k}{k+1} \beta_{k l}\left(\frac{\phi}{\pi R^{2}}\right)^{k+1}\right]+\left.\frac{\partial \alpha^{*}}{\partial V} \frac{\partial}{\partial \alpha} F\right|_{\alpha=\alpha^{*}}
\end{aligned}
$$

On the other hand, the pressure is directly calculated from the equilibrium state of our simulation as

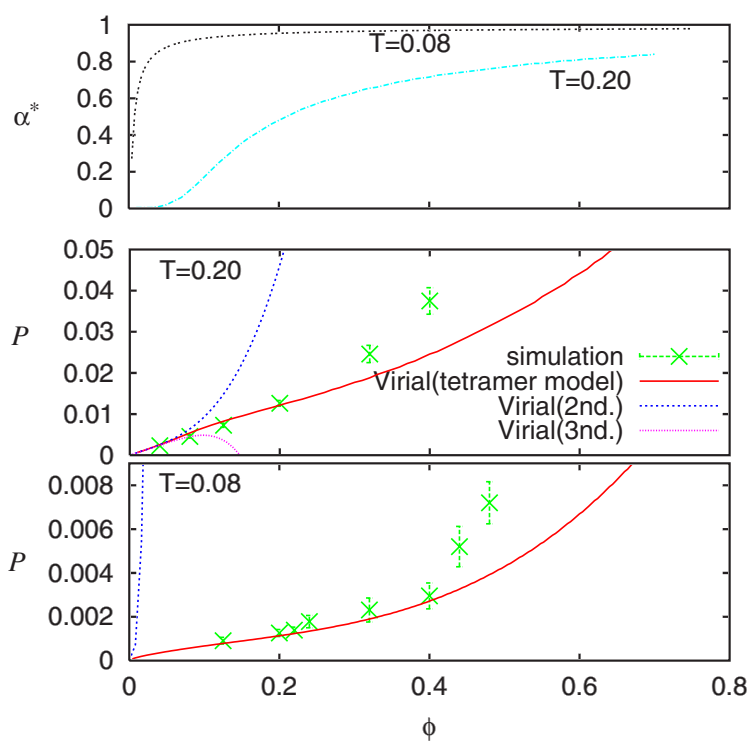

FIG. 10. (Color online) Optimized value of $\alpha$ and the pressure curves against $\phi$ are shown. Our tetramer model analysis (second order) agrees much better with the simulation compared to the standard virial expansion.

$$
\tilde{P}=\frac{1}{L^{2}}\left\langle N k_{B} T+\frac{1}{2} \sum_{i<j}^{N} \boldsymbol{r}_{i j} \cdot \boldsymbol{F}\left(\boldsymbol{r}_{i j}\right)\right\rangle .
$$

Seeing Fig. 10, the virial expansion with tetramer model agrees with the simulation drastically better than the standard virial analysis up to higher density. Not only that, it shows a phase transition. The critical point is at

$$
\phi_{c}=0.21, \quad T_{c}=0.073,
$$

which comes closer to the simulation result $-T_{c} \simeq 0.058$. These facts suggest that the locally ordered structure of tetragonal configuration is essential for determining the thermodynamic phase.

We want to emphasize again that the critical clusters of the liquid phase have local configurations which very much resemble the long range order of the solid phase. This may give important suggestions as to why the liquid phase is absent. To make this point clear, another modeling to calculate free energy around solidification approximately is proposed (Fig. 9, right). In this model, the system is divided into the $N$ square cells of equivalent size. Two types of particles are alternately assigned into each cell. Each particle can move only within its cell.

Figure 11 shows Gibbs free energy around the gas-liquid transition calculated by both the tetramer model and the cell divided model. Pressure is set to be that at the critical point: $P_{c}=0.00043$. Bifurcation of the local minimum value at $T_{c}$ $=0.073$ is associated with the second order transition to the

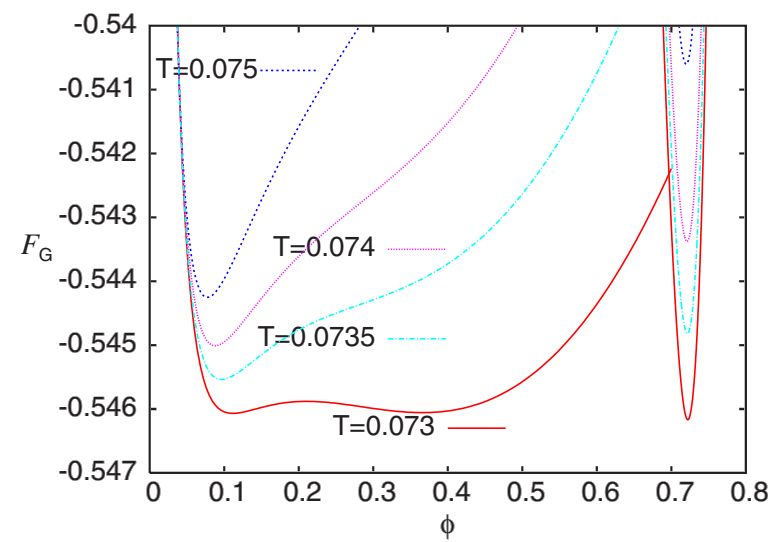

FIG. 11. (Color online) Gibbs free energy calculated by two kinds of models: the tetramer model (low density region) and the cell divided model (high density). 
gas-liquid phase. But almost at the same time, the minimum value of free energy switches to that of solid phase at higher density. It vividly illustrates the feature of two competitive kinds of transitions and a hidden liquid phase which are observed in our simulation.

\section{SUMMARY}

We have studied the binary monolayer of an Ising dipolar system with constant temperature particle dynamics simulation. At low temperature, particles constructed a solid cluster with tetragonal orientational order even at relatively low density, which is different from the result of dissipative (zero-temperature) simulations where various fractal clusters are observed.

At higher temperature, moderate aggregation into many finite sized clusters was observed, which was evidently shown to be the critical phenomena of condensation to a liquid cluster. The critical temperature of gas-liquid phase transition extrapolated from that cluster growth is, however, slightly lower than the temperature of solid phase, and thus it was concluded that liquid phase is absent in this system.

Detailed observation of the inner configuration of critical clusters clarified not only that the two point correlation function of them has a sharp peak at the contacting distance but that they locally have tetragonal symmetric configuration with alternate particles which very much resembles the long range order of the solid cluster. Though with the normal treatment of virial expansion we failed to explain the phase transition in this system, other trials of virial expansion improved by taking such alternate configuration into consideration succeeded in supporting simulation results quantitatively, showing competitive behavior of free energy between the critical transition of gas-liquid and solidification.

\section{DISCUSSIONS}

The universality class of the gas-liquid critical behavior is discussed here. Assuming that a particle has correlation with others within the cluster it belongs, the average cluster size defined in Eq. (5) is also described using the correlation function $g(r)$ as

$$
\frac{1}{\phi} \sum_{s} s^{2} n(s) \sim \int d r r g(r),
$$

which corresponds to the susceptibility $\chi$ in on-site spin models. In the two-dimensional Ising model, the value of exponents are known to be $\gamma=7 / 4$ and $\nu=1$. So, from the values of our estimation in Eqs. (7) and (11), it is concluded that this system belongs to the two-dimensional Ising universality class.

The most characteristic feature of this system is that there is no liquid phase. Disappearance of liquid phase has already been shown theoretically even in a simple fluid using an artificial pair potential model (potential with double Yukawa form) [22]. They pointed out that the fluid phase is gradually suppressed and finally disappears as the range of the attraction is reduced. But quantitatively, the parameters in [22] are not suitable for our system. Their criterion of no liquid phase is that the ratio of attractive interparticle range to the repulsive range is approximately less than 1.5 . That range is too short compared to the dipole interaction, so absence of liquid phase in our system cannot be simply due to the range of interaction.

We found that the key to controlling the behavior of condensation is the tetragonal configurations with alternate types of particles, which were already observed in critical clusters of liquid, and also determined the long-range order of the solid phase, reflecting the minimum energy configuration of this system. It can be said, in other words, that though the interaction of a pair potential is isotropic in this system, anisotropic interaction between the clusters (dimers tetramers and so on) rising from binary configuration dominates the characteristics of phase transitions.

Recently, this kind of disappearance of the critical transition was also reported in a similar binary particle system [23]. In a charged colloidal system modeled as hard spheres with point charge $+Q q$ and counterions of dimensionless point charge $-q$ (a three-dimensional system), it is reported that the critical transition disappears when the relative charge satisfies $20 \leq Q$. In our model, such liquid disappearance occurred even at $\mu_{\uparrow} / \mu_{\downarrow}=1$. It may be partly caused by the difference of the interaction range $\left(1 / r^{3}\right.$ instead of $\left.1 / r\right)$. The other difference is that both species in the IDP model have core diameter, and characteristic local structures due to those exclusive volumes played some important roles in phase transitions.

Future investigation will explain the reason for no liquid phase more clearly by trying simulation under other conditions of binary Ising dipolar systems, such as another relative dipole moment $\left(\mu_{\uparrow} / \mu_{\downarrow} \neq 1\right)$ and/or relative concentration $\left(\phi_{\uparrow} / \phi_{\downarrow} \neq 1\right)$. Because with those changes, the ground state configuration switches to others like hexagonal configuration (also refer to basic configurations I-V in [17]), thus it should change the feature of critical condensation and solidification.
[1] P. I. C. Teixeira, J. M. Tavares, and M. M. Telo da Gama, J. Phys.: Condens. Matter 12, R411 (2000).

[2] M. J. Stevens and G. S. Grest, Phys. Rev. E 51, 5962 (1995).

[3] J. M. Tavares, J. J. Weis, and M. M. Telo da Gama, Phys. Rev. E 73, 041507 (2006).

[4] J. M. Tavares, M. M. Telo da Gama, and M. A. Osipov, Phys. Rev. E 56, R6252 (1997).
[5] T. Tlusty and S. A. Safran, Science 290, 1328 (2000).

[6] P. J. Camp, J. C. Shelley, and G. N. Patey, Phys. Rev. Lett. 84, 115 (2000).

[7] J. C. Shelley, G. N. Patey, D. Levesque, and J. J. Weis, Phys. Rev. E 59, 3065 (1999).

[8] J. Stambaugh, D. P. Lathrop, E. Ott, and W. Losert, Phys. Rev. E 68, 026207 (2003). 
[9] D. L. Blair and A. Kudrolli, Phys. Rev. E 67, 021302 (2003).

[10] J. J. Weis, Mol. Phys. 100, 579 (2002).

[11] W. Wen, F. Kun, K. F. Pál, D. W. Zheng, and K. N. Tu, Phys. Rev. E 59, R4758 (1999).

[12] W. D. Ristenpart, I. A. Aksay, and D. A. Saville, Phys. Rev. Lett. 90, 128303 (2003).

[13] I. Varga, H. Yamada, F. Kun, H.-G. Matuttis, and N. Ito, Phys. Rev. E 71, 051405 (2005).

[14] I. Varga, F. Kun, and K. F. Pál, Phys. Rev. E 69, 030501(R) (2004).

[15] N. Yoshioka, I. Varga, F. Kun, S. Yukawa, and N. Ito, Phys. Rev. E 72, 061403 (2005).

[16] Y. Levin, Phys. Rev. Lett. 83, 1159 (1999).
[17] I. Varga and F. Kun, Philos. Mag. 86, 2011 (2006).

[18] S. Fazekas, J. Kertész, and D. E. Wolf, Phys. Rev. E 68, 041102 (2003).

[19] D. J. Evans and G. P. Morriss, Phys. Lett. 98A, 433 (1983).

[20] H. Watanabe, S. Yukawa, Y. Ozeki, and N. Ito, Phys. Rev. E 69, 045103(R) (2004).

[21] J. E. Mayer and M. G. Mayer, Statistical Mechanics (Wiley, New York, 1940), Chap. 12, p. 13.

[22] C. F. Tejero, A. Daanoun, H. N. W. Lekkerkerker, and M. Baus, Phys. Rev. Lett. 73, 752 (1994).

[23] A.-P. Hynninen and A. Z. Panagiotopoulos, Phys. Rev. Lett. 98, 198301 (2007). 